Revue d'histoire de l'Amérique française

REVUE D.HISTOIRE DE L'AMÉRIQUE FRANÇAISE

\title{
Essai sur les finances canadiennes (1700-1750)
}

\section{Guy Frégault}

Volume 12, numéro 3, décembre 1958

URI : https://id.erudit.org/iderudit/301916ar

DOI : https://doi.org/10.7202/301916ar

Aller au sommaire du numéro

Éditeur(s)

Institut d'histoire de l'Amérique française

ISSN

0035-2357 (imprimé)

1492-1383 (numérique)

Découvrir la revue

Citer cet article

Frégault, G. (1958). Essai sur les finances canadiennes (1700-1750). Revue

d'histoire de l'Amérique française, 12(3), 307-322.

https://doi.org/10.7202/301916ar d'utilisation que vous pouvez consulter en ligne.

https://apropos.erudit.org/fr/usagers/politique-dutilisation/ 


\section{ESSAI SUR LES FINANCES CANADIENNES}

\section{$(1700-1750)$}

\section{LES PROBLEMES - LA DOCUMENTATION}

L'histoire des finances du Canada sous le régime français présente un intérêt considérable. On l'a malheureusement fort peu étudiée. En 1887, l'archiviste Joseph Marmette portait le jugement suivant sur les registres des Archives des Colonies qui contiennent une partie des états financiers de la NouvelleFrance: «...II est aisé de se convaincre de prime-abord [sic] de leur manque d'intérêt au point de vue de l'histoire. » On possède déjà, poursuivait-il, les dépêches dans lesquelles les administrateurs de la colonie rendaient compte, tous les ans, de leur politique financière. "Chacune de ces lettres ... donne un aperçu plus que suffisant de la question pour nos futurs économistes qui seraient tentés de traiter ce côté de notre histoire; et je suis bien sûr de ne rien mettre sous le boisseau qui soit de nature à éclairer personne, en rejetant complètement dans l'ombre les 7 volumes que je viens de mentionner. ${ }^{1}$

Il n'est quand même pas interdit de chercher à tirer parti des registres que l'archiviste du siècle dernier rangeait si soigneusement dans les ténèbres extérieures. Non pas qu'ils apportent des réponses à toutes les questions que les «économistes》 peuvent se poser sur l'évolution du Canada avant la Conquête. L'historien de l'économie canadienne devrait dépouiller une docu-

1 (Rapport sur les archives françaises », Rapport sur les Archives du Canada 1887 (Ottawa, 1888), ccv. 
mentation infiniment plus étendue que celle-là. ${ }^{2}$ D'autre part, même le travailleur avant tout soucieux d'histoire générale ne peut pas se dispenser de prendre contact avec l'histoire financière. Celle-ci repose sur une documentation qui, malgré ses lacunes, est d'une richesse singulière. Parmi les indications qui s'en dégagent, les plus précieuses semblent bien être celles qui sont propres à expliquer la nature et les méthodes de la colonisation française telle qu'elle s'est déployée dans la vallée du Saint-Laurent et les territoires qui en dépendaient.

La colonisation française créa le Canada. Mais comment, au moyen de quelles ressources et à quelles fins ? Sans suffire à révéler tout cela, les données financières de la politique coloniale fournissent des précisions utiles en ce qu'elles viennent à propos compléter ce que des recherches ont commencé à mettre en lumière dans d'autres domaines, mieux explorés, de la vie de la Nouvelle-France. Il me paraît significatif que la meilleure étude dont nous disposions sur les finances de la collectivité canadienne se retrouve dans le beau livre d'Émile Salone. ${ }^{3}$ Celui-ci a bien compris qu'il eût été impossible de dégager les aspects essentiels de la colonisation de la vallée laurentienne sans savoir, au moins en gros, combien cette œuvre avait coûté à la métropole et comment cette dernière avait subvenu à la dépense. La documentation de Salone est sommaire et son étude, trop rapide. Cependant, parce que l'auteur aborde la question financière comme il aborde toutes les autres questions, - en mettant le problème de la colonisation au centre de ses préoccupations, -

2 Il lui faudrait, comme nous avons dû le faire pour cet essai, parcourir les registres de la série $A C, F 1$ et étudier en outre ceux de la série $A C$, F 2A (comportant quelques cartons remplis de pièces sur les compagnies de commerce), ceux de la série AC, F 2B (commerce des colonies) et ceux de la série AC, F 2C (contenant quelques statistiques d'importation et d'exportation). Il aurait le plus grand tort de négliger la série $\mathrm{AC}, \mathrm{C} 11 \mathrm{~A}$, y compris les vol. 113 à 119, auxquels Marmette fait allusion dans le texte cité plus haut. A vrai dire, il est essentiel de dépouiller tous les volumes de cette dernière série; il serait ridicule de les * rejeter complètement dans l'ombre » sous prétexte qu'ils ne contiennent que des pièces officielles. Il convient aussi d'explorer soigneusement la série AC, B. Les recensements du Canada sont conservés dans la série AC, G 1 et regorgent de données extrêmement suggestives. Il serait bon de compléter l'examen de ces grandes séries par celui des livres de comptes d'importantes institutions privées.

3 La Colonisation de la Nouvelle-France (Paris, [1906]), 411-430. 
ses conclusions, dans leur ensemble, demeurent valables, bien que, disposant maintenant d'une base documentaire un peu moins étroite, on puisse les clarifier, les nuancer et les approfondir.

Propres à jeter un jour nouveau sur la question capitale de la colonisation, des recherches sur les finances peuvent aussi apporter une contribution non négligeable à l'histoire économique et sociale de la colonie.

Qu'ajouteront-elles à nos connaissances d'histoire économique ? Remarquons d'abord, bien que ce soit superflu, que ce n'est pas dans les états financiers qu'il faut chercher des renseignements quantitatifs sur la production du pays. En ce qui concerne, par exemple, les denrées agricoles, ces précisions se trouvent ailleurs: dans les recensements qui, jusqu'à 1739 , révèlent les quantités de blé et d'autres céréales, de chanvre, de lin, de tabac, récoltées dans les seigneuries canadiennes, ainsi que le nombre de bestiaux qu'on y élève. Par ailleurs, les bordereaux de recette et de dépense fourmillent de données sur les prix: prix du blé, ${ }^{4}$ de la farine, du pain, des viandes, des vins, des alcools, des tissus, $\mathrm{du}$ fer, des huiles de poisson, des bois de construction, du bois de chauffage, etc. Naturellement, on y relève les quantités de vin et d'eau-de-vie que le pays importe de France et des Antilles, puisque des droits d'entrée frappent ces articles. On peut donc établir la valeur approximative de ce que les Canadiens produisent et consomment annuellement; la valeur de ce qu'ils transportent aussi, puisqu'on a les taux de fret entre Québec et Montréal, entre Montréal et les établissements du sud et de l'ouest.

Surtout, on découvre dans ces documents un grand fait économique: l'importance de l'État considéré comme client de l'industrie et du commerce locaux. Que l'Etat achète beaucoup et paye bien, aussitôt la prospérité se répand dans la collectivité canadienne; mais qu'il restreigne ses achats, pratique une économie rigoureuse, retarde trop ses paiements ou s'avise de ne pas honorer une partie de ses obligations, alors le pays éprouve du malaise, son commerce languit, et tout le monde crie misère.

${ }^{4}$ W. B. Munro a dressé un tableau des prix du blé dans The Seigniorial System in Canada (Cambridge, Mass., 1907), 94. Il y aurait intérêt à le rectifier à la lumière des bordereaux de dépenses. 
C'est que la balance des comptes est habituellement défavorable au commerce canadien. La colonie achète de la métropole et du reste de l'empire plus qu'elle ne leur vend; ce qu'elle vend au roi doit lui permettre de combler à peu près ce déficit. En 1736, par exemple, les importations canadiennes se chiffrent par 1.914.154 livres ${ }^{5}$ et les exportations, par 1.677.699. Cette dernière somme se compose comme suit: ${ }^{6}$

Denrées vendues à l'extérieur

334.332 livres

Pelleteries (autres que le castor) 491.240 “

Castor (vendu à la Compagnie des Indes) 381.367 “

1 navire construit pour des armateurs de Rouen 50.000 “

Lettres de change sur la Marine

Total

$\frac{420.760 \text { “ }}{1.677 .699 \text { “ }}$

Les dépenses du roi, qui se traduisent par le montant des lettres de change sur la Marine, renforcent d'autant le pouvoir d'achat du Canada, qui a besoin de soutien.

S'il est indispensable pour l'équilibre de l'économie canadienne que l'Etat soit un client assidu, il l'est également qu'il paye rubis sur l'ongle. D'où la nécessité absolue d'un solide crédit public. Crédit public implique évidemment monnaie. Sur les problèmes monétaires de la colonie, il existe, personne ne l'ignore, un excellent recueil de documents copieusement annotés et accompagnés d'un précis qui leur sert d'introduction; il s'agit de la compilation d'Adam Shortt. ${ }^{7}$ Malgré sa belle tenue, cet ouvrage est plus fécond en développements techniques qu'en explications proprement historiques. Il n'a certes pas échappé à l'auteur que les questions de monnaie se rattachent à des situations de finances, - le titre de son livre en fait foi, - mais il n'a pas assez dégagé les liens qui les articulent les unes aux autres. Le

\footnotetext{
5 \& Domaine d'Occident Année 1736. Extrait des marchandises venues de France, l'Isle Royale, et des Isles Françoise de L'amerique en l'année mil sept cent trente six suivant les Declarations qui en ont êté faites au Bureau du Domaine d'occident a Quebec », AC, C 11A, 121: 139-146.

6 \& Extrait des Denrées et Pelleteries Sorties de Canada et Lettres de Change en Retour des marchandises venues de france en lad. année mil sept cent trente six $», A C, C$ 11A, 121: 146v-147.

7 Documents relatifs à la monnaie, au change et aux finances du Canada sous le régime français (2 vol., Ottawa, 1925).
} 
simple fait qu'il ait estimé légitime de parler finances sans dépouiller d'états financiers nous force à conclure à une lacune considérable dans sa documentation, tout en nous mettant à même de saisir pourquoi ses analyses vont moins loin et ne portent pas aussi juste qu'elles auraient pu. Dans son domaine, Shortt a procédé un peu à la façon d'un démographe qui négligerait les recensements. Il faut, en conséquence, soumettre à un nouvel examen les réalités monétaires du régime français, sans néanmoins reprendre tout le travail de Shortt, travail bien fait, mais qu'on souhaiterait plus complet.

Ici comme ailleurs, l'histoire sociale apparaît liée à l'histoire économique. De même que les états financiers fournissent une foule de données susceptibles d'être exploitées pour poser les bases d'une histoire des prix, de même ils contiennent de quoi esquisser une histoire des revenus de quelques classes de la société. D'abord, on y voit les traitements des fonctionnaires, et donc les noms de ceux qui les touchent régulièrement, leur hiérarchie et leur nombre. Sur ce dernier point, ils offrent l'occasion de corriger l'impression, répandue par des historiens, que les fonctionnaires seraient «aussi peu nombreux que possible ${ }^{8}{ }^{8}$ S'ils ne sont pas anormalement nombreux, les serviteurs de l'État constituent un personnel relativement important et beaucoup mieux organisé qu'on ne le croirait. On pense ici à l'administration civile. Il ne faut pas oublier l'administration militaire. L'armée absorbe une bonne partie du budget des dépenses, et son entretien occasionne la formation d'un corps d'officiers qui, sans avoir, comme en Louisiane, l'allure d'une caste, a sûrement celle d'une aristocratie locale. Les fils de toutes les principales familles s'engagent dans la carrière des armes, et les familles qui « ont le plus de relief » constituent, ainsi que Bougainville l'a remarqué, un groupe distinct, dont des mariages soigneusement assortis resserrent les liens. ${ }^{9}$ Les militaires sont les mieux payés des serviteurs du roi : un simple capitaine touche une solde

${ }^{8}$ La Civilisation de la Nouvelle-France (Montréal, 1944), 157. J'avoue mon erreur.

9 《Mémoire sur l'état de la Nouvelle-France (1757) 》, Rapport de l'Archiviste de la province de Québec [RAPQ] pour 1923-1924 (Québec, 1924), 61s. 


\section{6 fois plus forte que les appointements d'un conseiller ordinaire au Conseil supérieur.}

L'étude des finances aide aussi à mieux comprendre la situation sociale du clergé et des services publics qui relèvent de lui. Non pas qu'on puisse calculer aisément tous les revenus de ce corps. A combien s'élèvent-ils ? Personne ne le sait au juste. L'État insiste longtemps pour que les institutions ecclésiastiques déclarent leur avoir et leurs charges. Il n'obtiendra jamais entière satisfaction. ${ }^{10}$ On sait cependant que, vers la fin de l'épiscopat de Saint-Vallier, le clergé du Canada dispose de ressources qui lui produisent au moins 120.000 livres par an. ${ }^{11}$ Il possède de vastes propriétés ${ }^{12}$ inégalement mises en valeur, perçoit les dîmes, détient des placements en France et retire des rentes constituées dans la colonie. ${ }^{13}$ Fait à souligner, il émerge réguliè-

10 Dupuy à Maurepas, 21 octobre 1726, AC, C 11A, 48: 298v; id. à id., 20 octobre 1727, AC, C 11A, 49: 274v; Aigremont à Maurepas, 15 octobre 1728, AC, C 11A, 50: 170; Maurepas à Dupuy, 18 mai 1728, ibid., 481481v; Hocquart à Maurepas, ler octobre 1731, AC, C 11A, 55: 41; Beauharnais et Hocquart à Maurepas, 5 octobre 1732, AC, C 11A, 57: 67v-68v.

11 Mémoire des biens de l'évêque, du chapitre et des autres communautés du Canada », H. Têtu et C.-O. Gagnon, éd., Mandements, lettres pastorales et circulaires des évêques de Québec (6 vol., Québec, 1887-1890), 1: 514-522. Cf. Saint-Vallier, «1725. Memoire du revenu de touts Les Etablissements Ecclesiastiques et Religieux de Canada \$, AC, C 11A, 47 : 426-427. L'évêque avoue à plusieurs reprises ne posséder que des renseignements incomplets, particulièrement dans le cas du revenu des Jésuites, aussi discrets, semble-t-il, envers lui qu'envers l'Etat. En additionnant les sommes qu'il donne pour toutes les institutions, on obtient le chiffre de 66.500 livres, évidemment trop bas. Par exemple, selon l'évêque, les religieuses de l'Hôtel-Dieu de Montréal ont un revenu de 3.000 livres; ellesmêmes en déclarent un de 10.000 (voir plus loin, note 13).

12 En 1734, les abbés de Brisacier et Tremblay estiment les propriétés du Séminaire de Québec à 200.000 livres, sans parler du terrain que cette maison possède dans la capitale. Auguste Gosselin, L'Eglise du Canada depuis Mgr de Laval jusqu'à la Conquête (3 vol., Québec, 1911-1914), 2 : 247.

13 Voici un exemple des revenus d'une institution religieuse et de la composition de son portefeuille. En 1734, les Hospitalières et l'Hôtel-Dieu de Montréal ont 140.775 livres placées sur l'Hôtel-de-Ville de Paris à 2.5\%, ce qui leur fait un intérêt annuel de 3.519 livres 7 sous. Leurs placements au Canada (rentes diverses) se chiffrent par 50.013 livres à $5 \%$, pour former des intérêts annuels de 2.500 livres 13 sous. Leurs terres leur rapportent, année commune, 2.012 livres et les subventions de l'Etat, 2.000 livres. Ainsi, les quelque 10.000 livres que touchent annuellement les religieuses de l'Hôtel-Dieu proviennent de revenus ainsi répartis:

Placements en France 
rement au budget du Canada. L'État subventionne les maisons d'enseignement et les services hospitaliers; écoles et hôpitaux ne sont pas uniquement des œuvres de charité. L'État contribue même aux frais du culte; il le fait pour suppléer au rendement insuffisant de la dîme, qu'il interdit de prélever sur un pied supérieur au 26e. Ce qui frappe, c'est l'importance relative de ces subventions: elles comptent, semble-t-il, pour environ le tiers des revenus connus du clergé. ${ }^{14}$

Il arrive aux membres de l'aristocratie et aux institutions religieuses de vendre à l'Etat des denrées que leurs seigneuries produisent. Il ne faut quand même pas ranger tous ceux qui font un commerce occasionnel dans la classe des commerçants. C'est peut-être sur ces derniers que l'histoire financière projette la lumière la plus vive. Pour plusieurs années, et particulièrement entre 1730 et 1750 , les bordereaux annuels de recette et de dépense comportent le détail de tout ce que l'État colonial se procure sur place, avec le prix qu'il en paye et les noms de ses fournisseurs. Il devient ainsi possible de connaître l'identité d'une grande partie de ceux qui composent la bourgeoisie commerçante et même juger de la place qu'ils y occupent au volume des ventes qu'ils font au roi. Les bordereaux ne mentionnent sans doute pas tous les marchands de la colonie. Il peut s'en trouver à qui l'Etat ne donne pas de commandes; il serait cependant étonnant que ceux-ci fussent à la fois nombreux et importants. ${ }^{15}$

Enfin, tandis qu'ils n'offrent que quelques éléments permettant de conjecturer, et encore d'une façon globale, les revenus des

Revenus de terres

$20 \%$

Subventions de l'Etat

$20 \%$

《 Etat des Biens et Revenus des pauvres de l'Hotel Dieu de Montreal », AC, C 11A, 61: 149-150; \& Etat au vray des Biens et revenus de la Communauté des Religieuses hospitalieres de Montreal », ibid., 151-153.

14 Proportion approximative. Une analyse du « Mémoire des biens ... 》 cité plus haut (note 11) indique que, sur 120.000 livres, 40.000 proviennent de subventions. Cependant, en 1735, les institutions ecclésiastiques reçoivent de l'Etat 67.200 livres ( $*$ Pour les Depenses de Canada a payer sur le fond du Domaine d'occident $1735 \gg, A C, F$ 1, 32: 82).

15 Un travail en cours au séminaire d'histoire de l'Université de Montréal permettra probablement de calculer le nombre approximatif des marchands du Canada au XVIIIe siècle. 
agriculteurs, les mêmes documents contiennent des indications utiles pour déterminer ce que peuvent gagner les artisans, les ouvriers et les manœuvres.

Le présent essai n'abordera pas tous les problèmes évoqués dans les pages qui précèdent. De plus, il limitera aux cinquante premières années du XVIII ${ }^{\mathrm{e}}$ siècle l'examen des questions qu'il retiendra. C'est que, pour la période antérieure à 1700 , la documentation est trop incomplète pour qu'on puisse retracer une ligne d'évolution même grossièrement approximative. Bien que la période suivante offre beaucoup plus de données, là encore, il y a des trous. $\mathrm{Si}$, par exemple, on possède le budget des dépenses de 1700 , on ignore le montant des débours effectués la même année. Ensuite, les chiffres manquent pour les années 1701-1704. Mieux partagé pour 1705, on peut utiliser le «Bordereau de la Recette et Depense faites en la nouvelle-france » cette année-là. Jusqu'à 1714 , force nous est de nous contenter de renseignements fragmentaires. Ensuite, les précisions se présentent plus nombreuses, quoique le chercheur doive s'accommoder de graves lacunes pour une période de quatre ans (1718-1721). Mais il disposera d'un excellent matériel à compter de 1722 et jusqu'à 1751. Après cette dernière date, il lui faut regarder ailleurs; il a atteint la période de Bigot, inaugurée en 1748 , et tout porte à croire que les états financiers de la colonie, pour les neuf dernières années du régime français, furent remis à la commission du Châtelet qui instruisit l'Affaire du Canada entre 1760 et $1763 .^{16}$

Non seulement pouvons-nous dépouiller une quantité croissante de documents à mesure que nous nous approchons du milieu du siècle, mais, point encore plus important, ces pièces deviennent de plus en plus détaillées. ${ }^{17}$ De 1730 à 1750, les bordereaux

\footnotetext{
16 Sur l'Affaire du Canada, voir le chapitre XVII de François Bigot, administrateur français (2 vol., Montréal, 1948), 2: 341-388.

17 Par exemple, \& l'Estat des fonds a faire pour les depenses de la nouvelle france pendant l'année $1700 \gg$ ne remplit que quatre pages; \& l'Estat des Depenses que Le Roy veut et ordonne estre faittes $\gg$ en 1707 en couvre quatorze. Le « Bordereau par extrait de la recette et depense contenüe au compte de 1732 » s'étend sur 24 pages. Le même bordereau remplit 44 pages en 1736,74 en 1739 et 90 en 1740 . En 1749, le \& Bordereau des Recettes et Dépenses 》 constitue un document de 120 pages.
} 
ne laissent à peu près rien à désirer. Le seul ennui est qu'il existe souvent plus d'un bordereau par année et qu'ils ne concordent pas dans tous les détails: signe du désordre qui règne dans les finances. (Ce désordre, fait révélateur, n'affligeait pas les contemporains; il provenait à la fois de mauvaises habitudes administratives et d'inconvénients inévitables, résultant des distances.) Il s'agit alors de comparer ces documents et de retenir ceux qui présentent les meilleures garanties d'exactitude. Mis bout à bout, les états qui résistent à la critique permettent de construire des séries chiffrées dont il me semble possible de tirer des conclusions utiles pour dégager avec clarté certaines des caractéristiques du régime français.

Les conclusions du présent travail ne peuvent être que partielles, vu que l'analyse ne portera que sur une partie - la plus importante, il est vrai, - des documents financiers. Ceux-ci se partagent en deux grandes catégories: les états «du roi » et les états «du Domaine», ainsi désignés parce que les dépenses comprises dans les premiers sont assignées sur les fonds du ministère de la Marine, alors que celles qui sont portées aux derniers sont assignées sur les fonds du Domaine d'Occident. ${ }^{18}$ L'état du roi, ai-je dit, est plus important que celui du Domaine. Il l'est d'abord en raison des sommes impliquées: un peu moins d'un demi-million au début du siècle, un peu plus vers 1730 et au delà de deux millions, en moyenne, à compter de 1743. Par comparaison, la somme à laquelle monte l'état du Domaine paraît modeste: de 1701 à 1715 , elle varie de 80.000 à 90.000 livres, pour osciller aux environs de 110.000 à 115.000 livres entre 1730 et 1750.

L'importance de l'état du roi relativement à celui du Domaine tient aussi à la nature des dépenses que l'un et l'autre prévoient. Les « charges » du Domaine sont fixes: appointements de l'étatmajor des trois gouvernements particuliers (Québec, Montréal et les Trois-Rivières), traitements des officiers de justice, subventions aux institutions religieuses, pensions et gratifications

18 Le fonctionnement de la ferme du Domaine - ce dernier fut rattaché à la Marine en 1732 - sera étudié dans un autre travail. La question est trop longue à débrouiller pour être traitée dans le présent essai. Voir provisoirement François Bigot, administrateur français, 2: 39s. 
diverses. Au contraire, la plupart des dépenses portées à l'état du roi varient avec les circonstances. Dans les années 1700, cet état comporte les chapitres suivants:

1. Entretien des fortifications et des bâtiments ;

2. Achat, construction et entretien de bateaux et de canots;

3. Courses et voyages;

4. Fret et voiture;

5. Gages des employés des magasins et des bureaux;

6. Loyers de maisons et de magasins ;

7. Journées d'ouvriers et façons d'ouvrages;

8. Présents aux sauvages ;

9. Bois de chauffage;

10. Hôpitaux ;

11. Achat de marchandises et de vivres;

12. Appointements des officiers généraux et autres entretenus;

13. Solde des troupes;

14. Gratifications ordinaires;

15. Gratifications extraordinaires ;

16. Dépenses imprévues. ${ }^{19}$

Les mêmes chapitres se retrouvent dans les comptes de 1749. Il s'y ajoute seulement celui des «préparatifs de guerre 》 (bien que la paix d'Aix-la-Chapelle vienne d'être conclue) et un coin fourre-tout étiqueté: «Autres diverses dépenses $\gg^{20}$ Il ne serait pas exagéré d'en conclure que, dès le commencement du XVIII ${ }^{\circ}$ siècle, l'état du roi a pris sa forme définitive.

Alors que l'état du Domaine intéresse seulement le clergé, une poignée de fonctionnaires et quelques pensionnés, l'état du roi

19 Dans les premières années du XVIIIe siècle, l'état du roi ne paraît compter que six chapitres; c'est qu'il réunit les onze premiers sous le seul titre de «Guerre et fortifications \$; chacun des chapitres que nous avons énumérés devient alors une section de cet unique chapitre. Voir l'état de 1700 (AC, C 11A, 113: 143-144v), celui de 1702 (AC, F 1, 10:228-229v) et celui de 1703 (AC, F 1, 11: 23-25). Un \& Estat des Depenses annuelles du Canada qui sont payées par le Tresorier de la Marine», préparé en 1715 (AC, C 11A, 113: 277-278), comporte les chapitres que j'ai énumérés dans le texte.

20 \& Etat des Payemens que le Roy veut et ordonne estre faits... Pour les depenses mentionnées cy apres faites et a faire pour le Service de Sa Majesté au pays de Canada pendant l'année 1749 », AC, C 11A, 116: 191200v. 
touche à tous les domaines de la vie coloniale et à presque toutes les classes de la société. Si l'histoire économique et sociale était l'objet de cet essai, il ne serait pas permis d'ignorer les états du Domaine. Mais une recherche sur tous les points mentionnés plus haut prendrait de trop vastes proportions; de plus, il me paraît que cette même recherche - particulièrement en ce qui concerne l'histoire des prix et celle des revenus provenant des ventes faites au roi - ne peut être menée à bien au moyen des procédés archaïques (fiches écrites à la plume, classification manuelle) qui demeurent habituels aux historiens et que ceux-ci auraient tort de se livrer, comme des artisans, à une besogne qu'un système mécanographique peut accomplir d'une façon plus sûre et plus expéditive.

En revanche, il est possible de mettre à profit les données que contiennent les états financiers, sans parler des explications que d'autres documents contemporains donnent à leur sujet, pour examiner de plus près comment - je veux dire de quelle manière et dans quel esprit - l'Etat métropolitain a mis en place son œuvre de colonisation économique au Canada. C'est le problème auquel on doit maintenant s'attaquer. On ne saurait toutefois l'aborder sans, en même temps, débroussailler le terrain, analyser certaines habitudes administratives et surtout construire quelques séries de chiffres. On voudrait qu'il ne parût pas excessif d'espérer qu'une telle ébauche puisse servir de point de départ à des travaux plus poussés, en attendant les synthèses nécessaires.

\section{L'ELABORATION DE L'ETAT DU ROI}

Ce serait, nous prévient-on, « un pur anachronisme » que de parler de «budgets » à l'époque qui nous occupe. On n'emploie pas encore le mot. La chose, cependant, n'est pas inconnue. ${ }^{21}$ Il existe des états annuels de prévision de recette et de dépense; il en existe pour le Canada. Chaque automne, au départ des vaisseaux à destination de la métropole, l'intendant expédie au ministre de la Marine ses prévisions pour l'année suivante. En

${ }^{21}$ Albert Duchesne, Histoire des finances coloniales de la France (Paris, 1938), 102. Voir ibid., 26v. 
France, les bureaux examinent son projet, le retouchent et le font approuver. Ce qui sort de cette machine administrative revient au printemps suivant à Québec sous le titre d'état du roi. Entre le budget élaboré au Canada et sa version retouchée par la Cour, il existe toujours des différences. Quelques exemples serviront à en indiquer les dimensions (Tableau I) .

\begin{tabular}{|c|c|c|c|c|}
\hline \multirow{2}{*}{\multicolumn{5}{|c|}{$\begin{array}{c}\text { TABLEAU I } \\
\text { ÉTATS DE PRÉVISION }\end{array}$}} \\
\hline & & & & \\
\hline \multirow{2}{*}{$\begin{array}{c}\text { Année } \\
1702\end{array}$} & \multicolumn{2}{|c|}{ Projet d'état } & \multicolumn{2}{|c|}{ Etat du roi } \\
\hline & \multicolumn{2}{|c|}{329.867 livres } & \multicolumn{2}{|c|}{303.654 livres } \\
\hline 1703 & 358.490 & “ & 332.462 & “ \\
\hline 1705 & 413.686 & “ & 326.255 & “ \\
\hline 1711 & 562.241 & “ & 388.632 & “6 \\
\hline 1713 & 448.373 & “ & 391.188 & “ \\
\hline 1717 & 350.545 & “ & 322.522 & “ \\
\hline 1719 & 292.465 & “ & 285.107 & “6 \\
\hline 1721 & 300.325 & “ & 309.543 & “6 \\
\hline 1722 & 299.433 & “ & 314.114 & “ \\
\hline 1723 & 309.243 & “ & 318.403 & $“$ \\
\hline 1724 & 294.817 & “ & 291.341 & “6 \\
\hline 1725 & 294.550 & $“$ & 289.696 & $" 6$ \\
\hline 1727 & 321.273 & “ & 308.156 & 6 \\
\hline 1729 & 394.288 & 6 & 306.098 & “6 \\
\hline 1731 & 354.430 & 6 & 334.425 & 6 \\
\hline 1732 & 379.499 & “ & 359.022 & “ \\
\hline 1734 & 363.874 & “ & 338.854 & “ \\
\hline 1741 & 445.637 & “6 & 323.625 & “ \\
\hline 1742 & 460.317 & “ & 426.488 & “ \\
\hline
\end{tabular}

Il ressort de ces chiffres que les bureaux de la Marine modifient invariablement les prévisions que leur envoie l'intendant de la Nouvelle-France. Ils les modifient d'ordinaire pour les diminuer: diminutions sensibles, puisque, pour la période qui précède la guerre de la Succession d'Autriche, les intendants ont 
réclamé, en moyenne, 367.000 livres par année, mais n'en ont obtenu que 332.000 , ce qui représente une réduction de $9 \%$.

On aura noté que, certaines années, le ministre de la Marine retranche beaucoup plus que $9 \%$ des prévisions de l'intendant. En 1711, par exemple, il les réduit de $30 \%$. Voici ce qui se passe à cette occasion. A l'automne de 1710, Raudot fait tenir à la Cour un état des dépenses extraordinaires que la guerre a provoquées. Les « dépenses faites en Canada en 1709, Sur les avis qu'on a eus que les anglois devoient venir l'ataquer» se chiffrent par 184.000 livres. ${ }^{22}$ De plus, en 1710 , les Canadiens ont envoyé des secours à l'Acadie et à Terre-Neuve et ils en ont reçu des prisonniers anglais, qu'il a fallu nourrir: le tout a coûté 30.600 livres. Voilà pour le passé. En raison de l'intensité qui peut marquer les opérations militaires en 1711 - ce sera l'année de Walker Raudot demande d'inscrire au budget 148.000 livres au chapitre "Guerre et fortifications». Il y a ensuite les frais ordinaires de l'administration: la solde des troupes, les appointements des officiers supérieurs, etc. En tout, pour assurer la défense du pays et le fonctionnement de ses services, l'intendant demande un peu plus de 560.000 livres. ${ }^{23}$ Maurepas ne l'entend pas de cette oreille. Il juge: «Les fonds extraordinaires qu'on demande icy pour le Canada me paroissent excessifs; il faut arranger cela s'il se peut et se mettre en regle. » Comment ? A coups de hache. Le ministre réduit à 90.000 livres (au lieu de 184.000) la somme réclamée pour régler les avances occasionnées par la guerre en 1709. Raudot a demandé 148.000 livres pour les onze premiers chapitres du budget: "Il n'y a ordinairement que $100 \mathrm{M}$ livres par an pour cette depense », observe Maurepas, qui n'en autorise effectivement que 100.000. Rien pour solder les dépenses provoquées en 1710 par les colonies atlantiques, que l'ennemi a d'ailleurs conquises. C'est ainsi qu'au lieu d'atteindre 562.000 livres, l'état du roi pour 1711 sera artificiellement ramené à 388.000 livres. $^{24}$

\footnotetext{
22 AC, F 1, 17: 49.

23 « Fonds demandez par M. Raudot Intendant en Canada pour 1711 》, ibid., 48.

24 \& Extraits des Comptes rendus par Les Commis des Tresoriers Generaux de la Marine a Quebec », AC, C 11A, 49: 369.
} 
Il faudra cependant que celui de 1712 soit haussé à 463.000 livres, dans une tentative d'absorber les excédents de dépenses des deux années précédentes. Tentative trop timide pour aboutir: une fois clos l'exercice de 1712, on s'aperçoit qu'on a laissé en souffrance pour 145.000 livres de dettes, qu'il faut rejeter sur 1713. Va-t-on les payer tout de suite ? Que non pas! L'état du roi retombe au-dessous de 400.000 livres, en 1713. Il ne prévoit que 60.000 livres pour régler (en partie) les créances de 1711 et de 1712. Mais, au moment où le roi donne à ses créanciers un début de satisfaction, il faut qu'il emprunte de l'autre main, car il reporte sur 1714 un excédent de dépenses de 130.667 livres. Et ainsi de suite. Après avoir dépouillé les états du roi depuis 1711 jusqu'à 1727, l'intendant Dupuy exprimait ainsi ses conclusions: "Que les depenses de la Colonie ont toujours êté plus fortes de beaucoup que les fonds faits par les etats du Roy. Que pour remplir ces depenses il a êté fait de tems a autre des Excedents de fonds ou remplacemens a la Colonie, sans quoy on n'eust pû la soutenir.» On ne saurait mieux décrire l'insuffisance systématique du budget.

Lorsqu'un intendant manque d'expérience ou qu'il a du caractère, ses prévisions manifestent infiniment plus de réalisme que celles de la Cour. Ce qui se produit en 1728 illustre bien ce point. Victime d'une destitution abrupte, Dupuy n'a pas le loisir de préparer le budget de 1729. Cette tâche retombe sur les bras de son successeur intérimaire, Clairambault d'Aigremont. Celuici profite de l'occasion pour démontrer au ministre de la Marine qu'au total, les budgets de 1726, 1727 et 1728 ont été inférieurs d'un bon demi-million aux dépenses effectuées au cours de ces trois années. Le fonctionnaire explique: «Ce n'est point sur le fondement qu'il y a eu precedemment des Excedents qu'il s'en trouve chaque année un considerable; cela provient de ce qu'on a toujours diminué le projet des fonds demandés pour les depenses de chaque année en supposant qu'il estoit trop fort. ${ }^{25}$ Il prépare, en conséquence, des prévisions qui s'élèvent à 394.000 livres; il n'y a employé, souligne-t-il, «que les depenses indispen-

25 Aigremont à Maurepas, 8 novembre 1728, AC, C 11A, 113: 347v. 
sables » et il «supplie » son chef de n'en rien retrancher. ${ }^{26}$ Le ministre n'en enlève pas moins 90.000 livres.

Hocquart arrive en 1729 , vers la fin d'août, trop tard pour veiller personnellement aux détails des prévisions de 1730 . L'année suivante, il prépare un budget dans lequel, souligne-t-il, il s'est appliqué à ne « comprendre que les dépenses qui sont indispensables suivant les connoissances qu'il en a prises par luy mesme ». ${ }^{27}$ Le résultat ? Un projet d'état sur lequel la Cour pourra se contenter de pratiquer une réduction de 20.000 livres. Les dépenses réelles se chiffreront, il est vrai, par 160.000 livres de plus que les prévisions de l'intendant. Il n'importe. Le consciencieux fonctionnaire n'aura pas eu le mauvais goût de proposer à son chef des dépenses excessives (il se sera borné à les faire). En transmettant à Hocquart le budget de 1731, Maurepas lui avait néanmoins fait observer: « Je suis persuadé que les fonds prévus dans l'état du roi seront plus que suffisants lorsque vous entrerés dans la vue d'oeconomie que Sa $\mathrm{Ma}^{\text {té }}$ desire et que je ne puis trop vous recommander. » Le ministre avait même ajouté qu'avec de telles disponibilités, « bien loin d'y avoir de l'excedent de dépense, il se trouvera du revenant bon $\gg{ }^{28}$ En 1740, Hocquart ne montrera pas la même prudence que dix ans plus tôt. Mieux instruit de la structure économique du Canada, il aura compris la nécessité de mises de fonds plus importantes de la part du gouvernement royal; la Cour, cependant, n'aura rien appris et elle diminuera de plus du quart les prévisions de son représentant.

En arrivant à Québec, en 1748, Bigot observe que le budget expédié de Versailles chaque année est fort insuffisant. Ce n'est pas, fait-il entendre à son chef, une économie que de fermer volontairement les yeux sur les dépenses à effectuer puisqu'elles se font quand même et qu'elles apparaissent ensuite, mais hors de saison, sous forme de comptes arriérés. ${ }^{29}$ Le système est toutefois trop bien établi pour que le magistrat puisse le changer.

26 Id. à id., 8 novembre $1728, \mathrm{AC}, \mathrm{C} 11 \mathrm{~A}, 50: 254-254 \mathrm{v}$.

27 Beauharnais et Hocquart à Maurepas, 24 octobre 1730, AC, C 11A, 113: $407 \mathrm{v}$; voir id. à $i d$., 25 octobre 1729 , Shortt, $2: 608$.

28 Maurepas à Beauharnais et à Hocquart, 1731, AC, C 11A, 56: 94-94v.

29 François Bigot, administrateur français, 1: 330. 
Il restera en vigueur jusqu'au dernier moment. En 1760, alors que les dépenses de l'année précédente avaient dépassé trente millions, le ministre de la Marine envoyait encore au Canada un budget invraisemblable de 2.400 .000 livres. ${ }^{30}$ Comme d'habitude, la Cour exigeait des économies. Elle croyait que le meilleur moyen d'en obtenir était de n'autoriser d'avance que des dépenses réduites et de laisser traîner les comptes. Obstinément fidèle à son système, quand la France se retirera de l'Amérique, elle laissera derrière elle une banqueroute. ${ }^{31}$

Le coup d'œil que nous venons de jeter sur l'élaboration du budget canadien nous met en mesure de conclure que l'état de prévision préparé à Québec sert à peine de guide au gouvernement français lorsque ce dernier arrête l'état du roi. Au contraire, la Cour tient à ce que les prévisions de l'intendant se modèlent sur les budgets qu'elle impose. D'où un écart constant entre les dépenses prévues et les dépenses réelles du Canada.

\section{(à suivre)}

GUY FrÉGaUlt

Université de Montréal.

${ }^{31}$ La Guerre de la Conquête (Montréal, 1955), 368-370. 